\title{
Existence of Limit Cycles for Liénard System with Application
}

Ali Bakur Barsham ALmurad, Elamin Mohammed Saeed Ali

Department of Mathematics. Faculty of Education. AL-Salam University.

E.Mail: alibakur1976@gmail.com

Department of Mathematics. Faculty of Education. Alzaem Alazhari University.

\begin{abstract}
This paper is part of a wider study limit cycle problems and planar system; The aims of this is to study the existence of limit cycle for Liénard system. We followed the historical analytical mathematical method to present a proof of a result on the existence of limit cycle for Liénard system form $\dot{x}=y-F(x)$,

$$
\dot{y}=-g(x)
$$
\end{abstract}

Key words: Liénard system, Limit cycles, existence and uniqueness.

Introduction: We are interested in existence and uniqueness of limit cycles for some polynomial planar systems. We discussed the existence by applying theorem1in [3]. We introduced the Liénard system and gave theorem for existence, uniqueness and amplitude of limit cycles. We applied the theorem for existence and uniqueness of limit cycles for some applications, and we verified the exactions, uniqueness.

\section{The Existence, Uniqueness of Limit Cycles:}

We consider the Liénard equation

$\dot{x}=y-F(x), \quad \dot{y}=-g(x)$

The above equation equivalent form:

$$
\ddot{x}+f(x) \dot{x}+g(x)=0
$$

Where $f(x)$ and $g(x)$ are continuous functions. This second order differential equation includes the famous Van der Pol equation.

$\ddot{x}+\mu\left(x^{2}-1\right) \dot{x}+x=0$

Define the functions:

$$
\mathrm{F}(\mathrm{x})=\int_{0}^{x} f(\mathrm{~s}) \mathrm{ds} \quad \mathrm{G}(\mathrm{x})=\int_{0}^{x} g(\mathrm{~s}) \mathrm{ds}
$$


Theorem1: Assume $F, g \in C^{1}(R), F(x)$ even, $g(x)$ odd, and $x g(x)>0$ for $x \neq 0$, if

(i) $F(0)=0, F(0)<0, F$ has single positive zero at $x=a$

(ii) $F$ increases for $x \geq a$ as $x \rightarrow \infty$

(iii) $G(x)=F(+\infty)=+\infty$.

Then the system (1) has exactly one limit cycle and it is stable.

\section{Some Applications:}

2.1. The following Liénard System

$$
\dot{x}=a x+b y+c x^{2}
$$

$\dot{y}=x+b x y+c x^{2}$

By using the Transformation

$$
\begin{array}{r}
\dot{z}=a z+b\left(x+b x y+c x^{2}\right)+2 c x z \\
\dot{z}=a z+b x+b^{2} x y+b c x^{2}+2 c x z
\end{array}
$$

For system (3)

$$
b y=\dot{x}-a x-c x^{2}=z-a x-c x^{2}
$$

$y=\frac{z-a x-c x^{2}}{b}$

Obtained (5) of (4)

$$
\begin{gathered}
\dot{z}=a z+b x+b^{2} x\left(\frac{z-a x-c x^{2}}{b}\right)+b c x^{2}+2 c x z \\
\dot{z}=a z+b x+b x z-a b x^{2}-b c x^{3}+b c x^{2}+2 c x z \\
\dot{z}=(a z+b x z+2 c x z)+\left(b x+b c x^{2}-a b x^{2}-b c x^{3}\right) \\
\dot{z}=(a+b x+2 c x) z+\left[b x+(b c-a b) x^{2}-b c x^{3}\right]
\end{gathered}
$$

replacing $z$ by $y$ we get

$\frac{d x}{d t}=y$,

$\frac{d y}{d t}=\left[b x+(b c-a b) x^{2}-b c x^{3}\right]+(a+b x+2 c x) y$

Or equivalently, to the type of

$$
\begin{gathered}
\frac{d x}{d t}=y-F(x) \\
\frac{d y}{d t}=-g(x) \\
F(x)=\int_{0}^{x} f(x) d x=\int_{0}^{x}(a+b x+2 c x) d x \\
F(x)=a x+\frac{1}{2} b x^{2}+c x^{2}=a x+\left(\frac{1}{2} b+c\right) x^{2}
\end{gathered}
$$


$\frac{d x}{d t}=y-\left[a x+\frac{1}{2}(b+2 c) x^{2}\right]$

$\frac{d y}{d t}=-g(x)=-\left[b x+(b c-a b) x^{2}-b c x^{3}\right]$

Where $F(x)=-\left[a x+\frac{1}{2}(b+2 c) x^{2}\right]$

The differential equation $f(x)=-(a+(b+2 c) x$

And $g(x)=\left[b x+(b c-a b) x^{2}-b c x^{3}\right]$

The integration function

$G(x)=\frac{1}{2} b x^{2}+\frac{1}{3}(b c-a b) x^{3}-\frac{1}{4} b c x^{4}$

Now we consider the first three focal values of (9) for system (8) by using the following result.

A quadratic system with a focus

$$
\begin{gathered}
\frac{d x}{d t}=a x-b y+a_{20} x^{2}+a_{11} x y+a_{02} y^{2} \\
\frac{d y}{d t}=b x+a y+b_{20} x^{2}+b_{11} x y+b_{02} y^{2}
\end{gathered}
$$

We get for $\mathrm{F}(\mathrm{x}) \quad a_{1}=a, \quad a_{2}=\frac{1}{2}(b+2 c)$,

We get for $g(x) \quad b_{1}=b, \quad b_{2}=b c-a b, \quad b_{3}=-b c$

In traduce the quantities.

For system:

$$
w_{1}=-a_{1}=-a,
$$

$$
\begin{gathered}
w_{2}=\frac{1}{8}\left(2 a_{2} b_{2}-3 a_{3}\right)=\frac{1}{8}\left[2\left(\frac{1}{2}(b+2 c)(b c-a b)-3(0)\right]\right. \\
w_{2}=\frac{1}{8}(b+2 c)(b c-a b) \\
w_{3}=c_{0}\left(6 a_{2} a_{4}+20 a_{4} b_{2}-15 a_{3} b_{3}\right)=0 \\
w_{1}<0, \quad w_{2} \neq 0 \text { in stable }, \quad w_{3}=0
\end{gathered}
$$

2.2. Now we considered the following Liénard system

$$
\begin{gathered}
\dot{x}=-y+m x+n x^{2} \\
\dot{y}=-x-n x^{2}-c x^{3}
\end{gathered}
$$

By using the Transformation

$$
\begin{aligned}
\dot{z} & =-\dot{y}+m \dot{x}+2 n x \dot{x} \\
\dot{z}=-\dot{y}+m z & +2 n x z
\end{aligned}
$$

For system (11)

$y=m x+n x^{2}-\dot{x}=m x+n x^{2}-z$

Obtain (13) of (12) 


$$
\begin{gathered}
\dot{z}=(m z+2 n x z)+\left(x+n x^{2}+c x^{3}\right) \\
\dot{z}=(m+2 n x) z+\left(x+n x^{2}+c x^{3}\right)
\end{gathered}
$$

Replacing $z$ by $y$ we get

$$
\begin{aligned}
& \frac{d x}{d t}=y, \\
& \frac{d y}{d t}=(m+2 n x) y+\left(x+n x^{2}+c x^{3}\right)
\end{aligned}
$$

Or equivalently, to the type of

$$
\frac{d x}{d t}=y-F(x)
$$

$\frac{d y}{d t}=-g(x)$

$$
\begin{gathered}
F(x)=\int_{0}^{x} f(x) d x=\int_{0}^{x}(m+2 n x) d x \\
F(x)=m x+n x^{2} \\
\frac{d x}{d t}=y-\left(m x+n x^{2}\right)
\end{gathered}
$$

$\frac{d y}{d t}=-g(x)=-\left(x+n x^{2}+c x^{3}\right)$

Where $F(x)=-\left(m x+n x^{2}\right)$

The differential equation $f(x)=-(m+2 n x)$

And $g(x)=\left(x+n x^{2}+c x^{3}\right)$

The integration function

$$
G(x)=\int_{0}^{x}\left(x+n x^{2}+c x^{3}\right) d x
$$

$G(x)=\frac{1}{2} x^{2}+\frac{1}{3} n x^{3}+\frac{1}{4} c x^{4}$

Now we consider the first three focal values of (17) for system (16) by using the following result.

A quadratic system (10) with a focus

We get for $\mathrm{F}(\mathrm{x}) \quad a_{1}=m, \quad a_{2}=n$,

We get for $\mathrm{g}(\mathrm{x}) \quad b_{1}=1, b_{2}=n, \quad b_{3}=c$

In traduce the quantities.

For system: $\quad w_{1}=-a_{1}=-m$,

$$
\begin{gathered}
w_{2}=\frac{1}{8}\left(2 a_{2} b_{2}-3 a_{3}\right)=\frac{1}{8}\left(2 n^{2}-3(0)\right)=\frac{1}{4} n^{2} \\
w_{3}=c_{0}\left(6 a_{2} a_{4}+20 a_{4} b_{2}-15 a_{3} b_{3}\right)=0
\end{gathered}
$$




$$
w_{1}<0, \quad w_{2} \neq 0 \text { in stable }, \quad w_{3}=0
$$

\section{References :}

Blows and N. Lloyd. The number of small amplitude limit cycles of Liénardequations, Math Proc. (1984).

Kuznets ov Y.A., Elements of Applied Bifurcation Theory, Applied Math. Sciences, (1995)

Jun L.D. , Xiang, et al. Bifurcation Theory and Methods of Dynamical systems [M]. Singapore: World Scientic Publ Co, (1997).

Lins, A. de Melo and C. Pugh. On Liénard equation. Lecture Notes in Math, Berlin/ New York, (1977).

Perko L. . Differential Equations and Dynamical Systems [M]. New York, Berlin, Heidelberg: Springer - Verlag, (2001).

Yan-Q Y. et al., Theory of Limit Cycles [J]. Trans. Math. Mono, Amer. Math. Soc. (1986). Zhilevich L.L., Some criteria for the absence of limit cycle and for the existence of a single limit cycle, Differential Equations, (1977). 